Review began 11/23/2021 Review ended 11/26/2021 Published 11/29/2021

(๑) Copyright 2021

Naqvi et al. This is an open access article distributed under the terms of the Creative Commons Attribution License CC-BY 4.0., which permits unrestricted use, distribution, and reproduction in any medium, provided the original author and source are credited.

\section{Hypertriglyceridemia-Induced Pancreatitis Complicated by Diabetic Ketoacidosis}

\author{
Syed Mujtaba Ali Naqvi ${ }^{1}$, Syed Haider ${ }^{1}$, Anup Patel ${ }^{1}$, Shoaib Muhammad ${ }^{2,3}$, Amman Yousaf ${ }^{4}$ \\ 1. Internal Medicine, University of Kentucky, Bowling Green, USA 2. Urology, Al-Aleem Medical College, Gulab Devi \\ Hospital, Lahore, PAK 3. Radiology, Salam Medical Complex, Lahore, PAK 4. Internal Medicine, McLaren Flint, \\ Michigan State University, Flint, USA
}

Corresponding author: Amman Yousaf, ayousafmd2@gmail.com

\begin{abstract}
Acute pancreatitis is a potentially fatal clinical entity having multiple underlying triggers. Though the incidence of hypertriglyceridemia-induced pancreatitis is low; however, patients with such risk factors develop severe disease. We present a case of a 47-year-old male who came to our facility with complaints of epigastric pain. Physical examination and laboratory workup unmasked the presence of pancreatitis alongside concurrent diabetic ketoacidosis (DKA). This presentation is unique, and to our knowledge, only a few cases have been reported in the literature. Furthermore, the co-existence of pancreatitis and DKA can overlap the clinical picture of each other, which might lead to unwanted complications if not diagnosed timely.
\end{abstract}

Categories: Endocrinology/Diabetes/Metabolism, Internal Medicine, Other

Keywords: diabetic ketoacidosis, insulin pump, hypertriglyceridemia-induced acute pancreatitis, pancreatitis causes, severe hypertriglyceridemia

\section{Introduction}

Acute pancreatitis (AP) is a life-threatening condition and a significant cause of acute abdominal pain. The incidence approaches up to 73 cases per 100,000; however, in the United Kingdom, the incidence range is 15-42 patients per 100,000 [1]. Out of a few crucial underlying etiologies, hypertriglyceridemia (HTG) poses a significant risk of developing severe disease. AP related to this pathology usually presents in predisposed patients having genetic lipoprotein abnormality. Such cases count around $2 \%-4 \%$ of all AP diagnoses. But HTG-induced pancreatitis is particularly important as it is associated with far more complications due to the severity [2]. We report a case of a 47-year-old male patient who presented with epigastric pain. The workup unmasked the presence of HTG-induced pancreatitis on the background of diabetic ketoacidosis (DKA). It is quite a rare presentation, and to our knowledge, only a few cases have been reported in the literature. Our article also emphasizes the importance of early diagnosis and management, as both AP and DKA can worsen each other.

\section{Case Presentation}

A 47-year-old male was transferred to the emergency department from a primary care facility with complaints of epigastric abdominal pain. In addition, his pain was associated with nausea and vomiting. On examination, the patient was in mild distress, and he was irritable. Palpation appreciated mild tenderness in the epigastric region; however, there was no guarding or rebound tenderness. His family history was insignificant for any lipid abnormalities, diabetes, or cardiac disease. His laboratory investigations revealed changes in blood glucose and bicarbonate, and urinalysis showed urinary ketones consistent with diabetic ketoacidosis. Significant laboratory value are summarised in Table 1.

\begin{tabular}{|c|c|c|c|}
\hline Parameters & Specimen & Value & Reference values \\
\hline Triglycerides & Serum & 8075 & $<150 \mathrm{mg} / \mathrm{dL}$ \\
\hline Blood glucose random & Serum & 278 & $72-99$ mg/dL \\
\hline Serum lipase & Serum & 5205 & $0-50 \mathrm{U} / \mathrm{L}$ \\
\hline Serum bicarbonate & Serum & 9 & 23-30 mEq/L \\
\hline Urinary ketones & Urine & $4+$ & Nil \\
\hline
\end{tabular}

TABLE 1: Laboratory investigations at the time of presentation 


\section{Cureus}

In line with increased triglycerides and lipase, a provisional diagnosis of hypertriglyceridemia-induced acute pancreatitis was made. To further evaluate, an urgent abdominal ultrasound was requested on which the pancreas couldn't be visualized due to overlying gassy bowel loops.

The patient was immediately admitted to the intensive care unit (ICU) and aggressive fluid resuscitation was done along with adequate analgesia and nutritional support. Parallel running DKA was managed with intravenous insulin with fluids and electrolytes replacement. He was also prescribed tablet fenofibrate 160 $\mathrm{mg}$ daily and tablet icosapent ethyl $2 \mathrm{~g}$ twice a day for overtly increased triglycerides. On the second day, the patient reported significant relief from abdominal pain, and his nausea and vomiting also settled. Laboratory investigations revealed a remarkable decrease in triglycerides to be $1508 \mathrm{mg} / \mathrm{dL}$ (Figure $1)$.

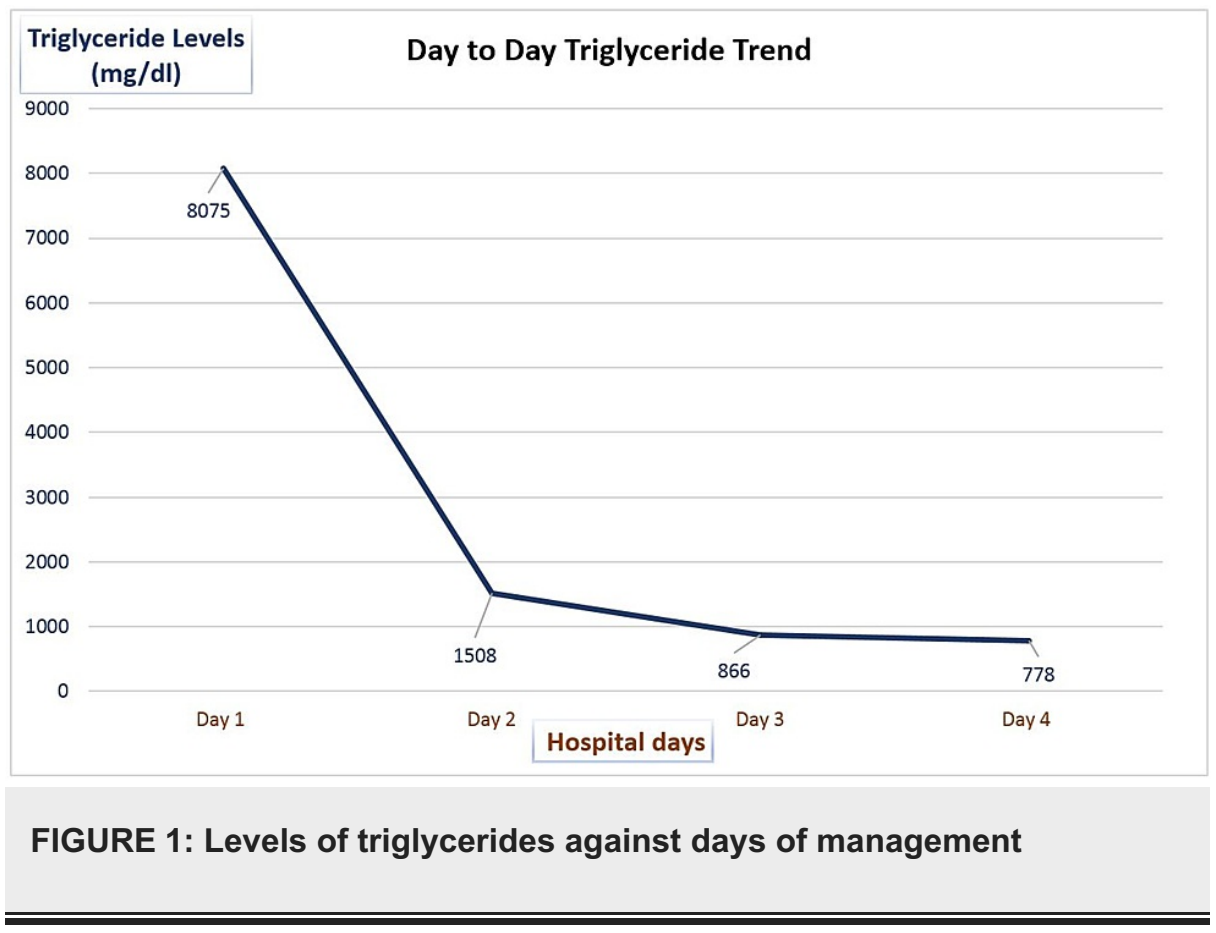

In addition, the anion gap closed back to normal, and metabolic acidosis resolved. Consequently, his insulin drip was stopped, and he was started on a combination of insulin glargine and lispro. He showed further improvements clinically, and his triglyceride level plummeted to $866 \mathrm{mg} / \mathrm{dL}$ on the next day. After the 36hour observance in the ICU, the patient was safely discharged on Tricor (fenofibrate), Vascepa (icosapent ethyl), atorvastatin alongside insulin Lantus (glargine) and Humalog (lispro). On one- and two-week followup visits, he showed no signs of relapse and is healthy to date.

\section{Discussion}

AP is a life-threatening inflammatory condition. After cholelithiasis and alcohol, HTG is the third most common trigger. Other noteworthy causes include direct pancreatic injuries, impaired secretion, and abnormally activated enzymes causing autodigestion of the pancreas. AP presents as severe abdominal pain, and laboratory investigations show elevated pancreatic enzymes in the blood.

HTG becomes a significant risk factor when the triglyceride level goes over $1000 \mathrm{mg} / \mathrm{dL}$. Various pathways are thought to play a part in the development of pancreatitis. Triglycerides are broken down into free fatty acids (FFAs) with the action of pancreatic lipases. Within the normal limits, these FFAs are bound by plasma proteins. In HTG, a significantly larger number of FFAs are released into the blood, outweighing plasma proteins' binding capacity. These unbound FFAs tend to bind together, forming smaller structures known as micelles. Due to their detergent properties, these micelles can damage the blood products and vessels, causing ischemia of the pancreas. Overtly increased FFAs also alter the blood properties like viscosity that ultimately leads to capillary blockage [3]. Consequently, ischemia of the pancreas results due to the loss of blood flow. Ischemia can eventually cause the activation of pancreatic enzymes by creating an acidic environment leading to autodigestion of the pancreas.

DKA is another potentially fatal state that results from a deficiency of insulin [4]. This causes decreased lipoprotein lipase activity, thereby worsening HTG. In type 2 diabetes, insulin resistance causes upregulation of triglyceride production. Concurrently, HTG-induced pancreatitis results in the reduced production of insulin in pancreatic beta cells [5]. Therefore, both pancreatitis and DKA overwhelm each other and worsen the clinical picture. 
Laboratory investigations play a vital role in the diagnosis and management of pancreatitis and DKA. The diagnosis of AP is based on the revised Atlanta classification criteria, which requires two out of the three conditions to be met: (i) acute onset of severe epigastric pain usually radiating to back, (ii) increased serum lipase and amylase to three times or more, (iii) findings of AP on imaging studies [6]. For HTG to be considered a culprit, a level of around $1000 \mathrm{mg} / \mathrm{dL}$ is needed. Our patient had two out of the three criteria for a diagnosis of AP. Furthermore, he had severely raised triglycerides to be considered the culprit. Similarly, DKA was diagnosed based on the laboratory findings of hyperglycemia and decreased bicarbonate levels.

Fluid resuscitation plays a pivotal role in managing both conditions, similar to the treatment of our patient. DKA was treated with intravenous insulin, and his electrolytes were normalized. Although no set guidelines on managing HTG-induced pancreatitis are developed, it is prudent to lower triglyceride levels. For this purpose, various strategies and medications are being used. We managed our patient with tablet Tricor (fenofibrate) and Vascepa (icosapent ethyl), and he showed excellent improvement in three days. Treating the concurrent DKA is also crucial, as decreased insulin causes a rise in the triglyceride level in the blood, creating a hindrance in the management of HTG. The use of insulin in such cases is bifold, as it manages DKA while also improving HTG by activating lipoprotein lipase [7].

\section{Conclusions}

Patients presenting with a suspected diagnosis of AP should also be evaluated for possible DKA and vice versa. As both the conditions worsen each other, it is crucial to manage them side by side. Laboratory investigations are vital in diagnosing, and imaging studies are critical in visualizing AP. Fluids play a pivotal role in managing HTG-induced AP as well as DKA. Triglyceride-lowering agents like fenofibrate are needed for HTG, while DKA should be treated with intravenous insulin.

\section{Additional Information \\ Disclosures}

Human subjects: Consent was obtained or waived by all participants in this study. Conflicts of interest: In compliance with the ICMJE uniform disclosure form, all authors declare the following: Payment/services info: All authors have declared that no financial support was received from any organization for the submitted work. Financial relationships: All authors have declared that they have no financial relationships at present or within the previous three years with any organizations that might have an interest in the submitted work. Other relationships: All authors have declared that there are no other relationships or activities that could appear to have influenced the submitted work.

\section{References}

1. Yadav D, Lowenfels AB: Trends in the epidemiology of the first attack of acute pancreatitis: a systematic review. Pancreas. 2006, 33:323-330. 10.1097/01.mpa.0000236733.31617.52

2. Wan J, He W, Zhu Y, et al.: Stratified analysis and clinical significance of elevated serum triglyceride levels in early acute pancreatitis: a retrospective study. Lipids Health Dis. 2017, 16:124. 10.1186/s12944-017-05173

3. Kimura W, Mössner J: Role of hypertriglyceridemia in the pathogenesis of experimental acute pancreatitis in rats. Int J Pancreatol. 1996, 20:177-184. 10.1007/BF02803766

4. Ahmad S, Yousaf A, Muhammad S, Ghaffar F: Coexistence of diabetic ketoacidosis and thyrotoxicosis: a jeopardy of two endocrine emergencies. BMJ Case Rep. 2021, 14:e243534. 10.1136/bcr-2021-243534

5. Reghina AD, Craciun S, Fica S: Severe transient hyperglycemia in a prediabetic patient during mild acute pancreatitis. Case Rep Med. 2015, 2015:968593. 10.1155/2015/968593

6. Banks PA, Bollen TL, Dervenis C, et al.: Classification of acute pancreatitis-2012: revision of the Atlanta classification and definitions by international consensus. Gut. 2013, 62:102-111. 10.1136/gutjnl-2012302779

7. Inayat F, Zafar F, Riaz I, Younus F, Baig AS, Imran Z: Hypertriglyceridemic pancreatitis: is insulin monotherapy a feasible therapeutic option?. Cureus. 2018, 10:e3461. 10.7759/cureus.3461 